\title{
Assessment of LV Function by Three-Dimensional and Three-Dimensional Speckle Tracking Echocardiography after Percutaneous Coronary Intervention for Chronic Total Occlusion Coronary Artery Disease \\ Kamal Ahmed Marghany Mahgoub \\ Department of Cardiology, Faculty of Medicine, Al-Azhar University, Egypt
}

\begin{abstract}
Background: Cardiovascular disease is the most leading cause of morbidity and mortality throughout the world today. Chronic total occlusions are defined as $100 \%$ occlusions with TIMI flow 0 with at least 3 -month duration. New echocardiographic techniques is an important noninvasive method for assessing cardiovascular function and mechanics.

Objective: Determination of the impact of revascularization for chronic total occlusion coronary artery disease on left ventricular function using three-dimensional and three- dimensional speckle tracking echocardiography. Patients and methods: The present study included (60) patients presenting with chronic total occlusion coronary artery disease at Al-Azhar University Hospitals, in the period between December 2015 and April 2018. The study compared the differences in echocardiographic parameters before the procedure (pre-procedure) and one month after successful PCI to CTO (post- procedure). Two-dimensional (2D) and three- dimensional (3D) transthoracic echocardiography (baseline and follow up study), 2D Global longitudinal strain (GLS) and 3D longitudinal strain (LS) speckle tracking echocardiography (base line and follow up study) were done.

Results: Mean left ventricular ejection fraction significantly increased, while the left ventricular end-diastolic and end-systolic volumes significantly decreased. The left ventricular end-systolic and end-diastolic volumes and diameters were significantly decreased. The global longitudinal strain (2DSTE and 3DSTE) showed significant improvement after successful revascularization. Both patients with LVEF $\geq 50 \%$ and LVEF $<50 \%$ displayed significant improvement in the 2D GLS and 3D LS, also diabetic and non-diabetic patients displayed significant improvement in the 2D GLS and 3D LS. Improvement in the 2D GLS \% was correlated with an improvement in the 3D LS \%.
\end{abstract}

Conclusion: Restoring the coronary blood flow in chronic total occlusion patients reduces the left ventricular volumes, dimensions and improves the left ventricular ejection fraction and global longitudinal strain of hibernating myocardium.

Keyword: Chronic Total Occlusions, Left Ventricular Function, New echocardiographic technique, Percutaneous Coronary Intervention.

\section{INTRODUCTION}

Cardiovascular disease is the leading cause of morbidity and mortality throughout most of the world today, representing $31 \%$ of all deaths ${ }^{(1)}$. Coronary artery disease is the most common cardiovascular disease and its incidence increases in the elderly ${ }^{(2)}$. Population aging and the increase of risk factors such as arterial hypertension and diabetes associated with obesity have greatly contributed to increased hospitalizations of patients with acute coronary syndrome (ACS) ${ }^{(3)}$. Coronary chronic total occlusions (CTOs) are defined as $100 \%$ occlusions with TIMI (Thrombolysis in Myocardial Infarction) 0 flow with at least a 3month duration (4). Echocardiography is an important noninvasive method for assessing cardiovascular function and mechanics (5). Recently, the use of two-dimensional speckletracking echocardiography (2D-STE) for measurement of myocardial strain has gained importance for its applicability in the clinical practice. Its high sensitivity to measure systolic function and identify left ventricular subclinical dysfunction, as compared with left ventricular ejection fraction, extends its applicability and makes it a test of additional value in many areas of cardiology ${ }^{\left({ }^{(}\right)}$. Despite promising data, 2D-STE has not been sufficiently standardized as a routine method for the diagnosis of myocardial ischemia. Characteristics inherent to the technique affect its applicability in both acute and chronic phase of the ischemic event, as previous ventricular deformities may affect the interpretation of results ${ }^{(7)}$. The study by dos Santos et al. ${ }^{(8)}$ provides us with a pioneer study on the real applicability of left ventricular longitudinal strain in UA. The authors described the frequency at which 2D-STE is indicated in cardiac emergencies and evaluated the values of the test in patients with severe coronary artery lesions. Two dimensional speckle tracking echocardiography is based on tracking the characteristic speckle patterns created by interference of ultrasound beams in the myocardium and its accuracy has been confirmed using son micrometry and magnetic resonance imaging as reference methods ${ }^{(9)}$. Measurements of $\mathrm{S}$ and SRs are more sensitive than standard 
echocardiographic parameters for assessing left ventricular (LV) function of many clinical conditions, including cardiomyopathy and $\mathrm{CAD}^{\left({ }^{(10)}\right.}$. Standard echocardiography has several targets in the cardiac population, as the assessment of myocardial performance, valvular and/or congenital heart disease, and hemodynamics. Three-dimensional echocardiography gained attention recently as a viable clinical tool in assessing left ventricular (LV) and right ventricular (RV) function, volume, and shape (11). The improved capability of real-time 3DE includes visualization of heart structures with depth, assessment of cardiac lesions from any perspective, and quantification of volumes without geometric assumption. The technology enables accurate, precise, detailed understanding of the pathophysiologic nature of diseases. 3DE data acquisition includes simultaneous multi plane mode, real-time 3D mode narrow sector, focused wide sector-zoom mode, full volume-gated acquisition, and full volume with color flow Doppler ${ }^{(12)}$. In 3DE, the LV end-diastolic volume (EDV) and end-systolic volume (ESV) are measured by semi-automatic border delineation in $3 \mathrm{D}$ space with manual adjustment when required. In cases where border delineation is still difficult owing to trabeculae or poorly defined with $3 \mathrm{DE}$ imaging, contrast agents maybe infused to enhance LV opacification. The 3DE eliminates geometric assumption or circumvents foreshortened views that are the common source of errors in 2DE. In addition, several studies have shown that LV mass determined by 3DE also correlated better with CMR compared to 2DE with $\mathrm{CMR}^{(\mathbf{1 3})}$. Three-dimensional speckle tracking echocardiography (3DSTE) has recently been regarded as a more promising technique to accurately and reproducibly evaluate the segmental and global LV function ${ }^{(14)}$.

\section{PATIENTS AND METHODS}

The present study was a prospective study included (60) patients presenting with chronic total coronary artery occlusion at Al-Azhar University Hospitals, in the period between December 2015 and April 2018. The study compared the difference in echocardiographic parameters between the pre procedure value and first month after successful PCI to CTO (post procedure). The study was approved by the Ethics Board of Al-Azhar University and an informed written consent was taken from each participant in the study.

Inclusion criteria: Patients with chronic total coronary artery occlusion which was defined as lumen compromise resulting in either TIMI flow grade 0 or 1 , with a likely duration of more than 3 months documented by history, clinical examination, resting electrocardiography, transthoracic echocardiography and coronary angiography. Patients who had successful percutaneous coronary intervention to chronic total occlusion.

Exclusion criteria: Patients who had failed percutaneous coronary intervention to chronic total occlusion. Any coronary lesions which not considered chronic total occlusion.

Methods: Informed consent was taken. Full history taking and examination to assess: Cardiovascular risk factors e.g. smoking, obesity, diabetes mellitus and hypertension that is defined as BP values $\geq 140$ $\mathrm{mmHg}$ SBP and/or $\geq 90 \mathrm{mmHg}$ DBP on 2 separate occasions and or on antihypertensive treatment regularly ${ }^{(15)}$.

Laboratory investigations: including complete blood picture, fasting blood glucose, HbA1c, serum lipogram, serum creatinine, liver function tests, and serology for hepatitis B and C.

Clinical Examination: Full clinical examination were carried out, chest and heart examination for heart sounds, additional heart sounds, murmurs and the back for lung congestion. Electrocardiography (ECG) were done by an investigator to diagnose chronic ischemic heart disease, using resting 12leads ECG.

Two-dimensional

transthoracic echocardiography (baseline study): Vivid E95 phased array system equipped with TDI, speckle tracking and three-dimensional technologies was used. Two dimensional transthoracic echocardiography was done before and one month after successful percutaneous coronary intervention. All the patients were examined in the left lateral decubitus position. Echocardiographic images were acquired from the standard views (parasternal long-axis, parasternal short axis, apical four-chamber, apical five-chamber and apical twochamber views) ${ }^{(16)}$. Left ventricular systolic function was assessed. Measurement of LV Ejection fraction $(\mathrm{EF} \%)$ and fractional shortening (FS\%) were done. Assessment of left ventricular systolic function two-dimensional echocardiography using the Simpson's method. In this method the left ventricle is divided into 20 sections of known thickness. The computer takes multiple short-axis slices at different levels. The volume of each slice is area multiplied by its thickness. LVEF \% was calculated as percentage change of LV chamber volumes between diastole and systole. The LV ejection fraction (EF \%) was automatically calculated as follows: $\mathrm{EF} \%=(\mathrm{EDV}-$ $\mathrm{ESV}) / \mathrm{EDV} \times 100^{\left({ }^{(17)}\right.}$

Two-dimensional speckle tracking (Global longitudinal strain): Generally two-dimensional ultrasonic detectors were used to collect images of 
the apical two-chamber, apical four-chamber and apical three-chamber views. During the inspection, all patients were connected to timing electrocardiography and maintained in the left lateral decubitus position. This involved entering two-dimensional speckle tracking mode, adjusting the baseline and angle, clearly displaying the image of the endocardium, mitral annulus and apex, and speckle assignment separately. The curve of the endocardium was drawn and the software analyzed the data and calculated the peak value longitudinal strain of 17 sections of the left ventricle and the time to peak value of the strain ${ }^{(18)}$.

\section{Three-dimensional transthoracic and 3D speckle} tracking echocardiography (baseline study): Three-dimensional transthoracic echocardiography was done before successful percutaneous coronary intervention. Real-time three-dimensional echocardiography images were obtained from an apical window with the patient in the same position as for two-dimensional speckle tracking echocardiography. Full-volume images were gathered over four cardiac cycles using a matrix array transducer. Measurements of the three dimensional-left ventricular volumes, the three dimensional ejection fraction and three dimensional speckle tracking were performed off-line. Recently, published EAE/ASE guidelines recommend the use of 3D echocardiography in assessing LV volumes and ejection fraction as a more accurate and reliable modality. Latest advances in speckle tracking echocardiography (STE) also allow us to track speckles in all three dimensions simultaneously in a full-volume data set ${ }^{(\mathbf{1 9})}$.

Coronary angiography: Transfemoral standard technique is used in all patients using $6 \mathrm{Fr}$ sheath. All procedures were performed using Siemens Axiom Arts and Philips Allura Xper FD 10/10. The procedures and lesions identifications were performed by expert interventional cardiologists.

1. Pre-procedural preparation: a) Informed consent: A detailed discussion with the patient and family for indications, benefits, potential complications, possibility to need emergency CABG as well as alternative options then an informed consent was taken. b) Patient should be fasting several hours before procedure. c) Medications: All medications are continued till morning of the procedure. Aspirin $325 \mathrm{mg}$ orally is given before the procedure. If the patient is not on Clopidogril $75 \mathrm{mg}$, a loading dose of $600 \mathrm{mg}$ PO is given prior to the procedure. d) Education: Patients were warned that they may feel nauseated or may experience a hot sensation lasting for about 30 seconds due to the injections of non-ionic contrast dye (Optiray. USA). Also, they are instructed to cough once they hear anyone in the room say "cough" which is a maneuver to accelerate resolution of dye-induced bradycardia. e) Equipment: i. Continuous blood pressure \& ECG monitoring of heart rate, rhythm \& ST segments during the whole procedure is essential. ii. Resuscitation equipment, in particular defibrillators $\&$ intubation trays, are always tested \& ready. 2 . PCI procedure: (a) Access site: Trans femoral standard technique is used in all patients using $6 \mathrm{Fr}$ sheath. (b) Intraprocedural medications: As soon as the arterial sheath is in place, a dose of 10.000 units of heparin is injected intravenously. If the procedure continues for more than 1 hour, another 5000 units of heparin is given. (c) Coronary angiography was performed assessing: CTO lesion as regards: 1. Site of the occlusion. 2. Length of the occlusion. 3. TIMI flow grade. 4. If calcified, degree of calcification. 5. Collaterals using Rentrop grade. 6. Presence or absence of bridging collaterals. TIMI Grade Flow: TIMI Grade Flow' is a scoring system from 0-3 referring to levels of coronary blood flow assessed during percutaneous coronary angioplasty: TIMI 0 flow (no perfusion) refers to the absence of any ante grade flow beyond a coronary occlusion. TIMI 1 flow (penetration without perfusion) is faint ante grade coronary flow beyond the occlusion, with incomplete filling of the distal coronary bed. TIMI 2 flow (partial reperfusion) is delayed or sluggish ante grade flow with complete filling of the distal territory. TIMI 3 is normal flow, which fills the distal coronary bed completely. Collaterals were graded by the degree of filling of the involved vessel beyond the CTO according to the Rentrop classification. Grade $0=$ no visible filling. Grade $1=$ side branch filling but without any dye reaching the epicardial segment. Grade 2 = partial epicardial filling. Grade $3=$ complete filling of the epicardial segment. Angiographic patterns outlining the morphologic characteristics of the vessels and defining lesion specific characteristics are identified to influence the likelihood of a successful PCI. Several projections were performed in order to better visualize the coronary arteries and overcome the problem of foreshortening and superimposition of the vessels. (d) Guiding catheter selection: For the left coronary system, extra backup type guiding catheters (extra backup) were preferred and used in most cases. (e) Guide wire selection: The wires used in this study were PT2 (moderate support), Gaia Second, Pilot 50 \& 150, Miracle 3 and Fielder XTA. (f) Strategies to open CTO: Antegrade approach to CTO was the strategy applied in all patients of our study. (g) After guide wire successfully cross the CTO, subsequent balloon angioplasty and stent implantation were performed with appropriate-sized devices. The first attempt to 
cross the CTO with a $1.5-2.0 \mathrm{~mm}$ diameter, low profile balloon with a tapered tip and a lubricious coating facilitate crossing even long lesions. All successfully opened CTO were treated with DES. (h) When we stop the recanalization procedure: When a CTO is proving refractory to initial maneuvers, the operator weight the likelihood of success with continued persistence against the risk of complications developing.

Two-dimensional and Three-dimensional
transthoracic and speckle tracking
echocardiography (follow up study) :
Reassessment of left ventricular function (LVEF\%), left ventricular end-diastolic and endsystolic dimensions (LVEDd \& LVESd) and also two dimensional speckle tracking (GLS) one month after successful PCI to CTO lesion.

\section{Statistical analysis of data}

Statistics were done by computer using The SPSS version 15.0 for Windows statistical software package program. The continuous variables are reported as the mean \pm standard deviations (SD) and the categorical variables are expressed as percentages. A comparison of the continuous variables pre and one month after the procedure was performed using paired samples test. The GLS and velocities were compared using paired samples test. The comparison between (2D \& 3D) speckle tracking pre-and post-procedure in diabetic and non-diabetic patients was performed using paired samples test. The comparison between 2D GLS pre and post procedure in patients with $\mathrm{EF} \geq 50 \%$ and patients with $\mathrm{EF}<50 \%$ was performed using paired samples test. The comparison between 3D LS preand post-procedure in patients with $\mathrm{EF} \geq 50 \%$ and patients with $\mathrm{EF}<50 \%$ was performed using paired samples test. Linear correlation between 2D GLS and 3D LS was performed using person correlation test. The delta values defined as the difference between the first month and the pre-procedure value. A value of $\mathrm{p}<0.05$ was considered statistically significant.

\section{RESULTS}

Demographic data and risk factors: Age of the patients ranged from 48 to 72 years with a mean of $60.8 \pm 6.2$ SD years [ 39 males $(65 \%)$ and 21 females (35\%)]. Hypertension: There were 38 hypertensive patients $(63.33 \%)$ and 22 non- hypertensive patients (36.67\%). The study included 32 patients with diabetes $(53.33 \%)$ and 28 patients were nondiabetics (46.67\%). 38 patients of our study population were smokers $(63.33 \%)$ and 22 patients were non- smokers (36.67\%) (Table 1).

Table (1) Incidence of risk factors in the study

\begin{tabular}{|c|c|c|}
\hline Risk factors & Number & Percentage \\
\hline Hypertension & 38 & $63.33 \%$ \\
\hline Diabetes mellitus & 32 & $53.33 \%$ \\
\hline Smoking & 38 & $63.33 \%$ \\
\hline
\end{tabular}

Echocardiographic parameters (pre and post procedure): Comparison between LVEF (\%) by Simpson's method pre and post procedure:

In pre procedure, LVEF by Simpson's method ranged from $41 \%$ to $75 \%$ with a mean of $57.0 \pm 8.7$ SD $(\%)$. In post procedure, ranging from $44 \%$ to $76 \%$ with a mean of $58.5 \pm 8.1 \mathrm{SD}(\%)$. There was significant increase in the mean LVEF \% measured by $2 \mathrm{DE}$ (Simpson's method) post procedure (Table 2).

Table (2): Comparison between LVEF \% by Simpson's method pre and post procedure.

\begin{tabular}{|c|c|c|c|c|c|c|c|c|c|c|}
\hline \multirow{2}{*}{ Time } & \multicolumn{4}{|c|}{ EF \% 2D } & \multicolumn{2}{c|}{$\begin{array}{c}\text { Paired } \\
\text { Differences }\end{array}$} & \multicolumn{2}{c|}{ Paired Samples Test } \\
\cline { 2 - 10 } & \multicolumn{2}{|c|}{ Range } & Mean & \pm & SD & Mean & SD & t & P-value \\
\hline Pre & 41 & - & 75 & 56.950 & \pm & 8.650 & -1.533 & 1.384 & -8.585 & $<0.001^{*}$ \\
\hline Post & 44 & - & 76 & 58.483 & \pm & 8.081 & & & & \\
\hline
\end{tabular}

There was very high statistically significant comparison between LVEF \% by 2 DE (Simpson's method) pre and post procedure with a $\mathrm{P}$ value $<0.001$.

In pre procedure, LVEDd ranged from $3.3 \mathrm{~cm}$ to 6.8 $\mathrm{cm}$ with a mean of $5.5 \pm 0.6 \mathrm{SD}(\mathrm{cm})$. In postprocedure, LVEDd ranged from $3.3 \mathrm{~cm}$ to $6.7 \mathrm{~cm}$ with a mean of $5.01 \pm 0.6 \mathrm{SD}(\mathrm{cm})$. There was significant decrease in the mean LVEDd $(\mathrm{cm})$ postprocedure. Highly Significant statistical comparison was seen between LVEDd $(\mathrm{cm})$ preand post-procedure with a $\mathrm{P}$ value $<0.001$. In pre procedure, LVESd ranging $2.1 \mathrm{~cm}$ from to $5.3 \mathrm{~cm}$ with a mean of $3.5 \pm 0.6 \mathrm{SD}(\mathrm{cm})$. In postprocedure, LVESd ranged from $2.1 \mathrm{~cm}$ to $5.1 \mathrm{~cm}$ with a mean of $3.5 \pm 0.6 \mathrm{SD}(\mathrm{cm})$. There was significant decrease in the mean LVESd $(\mathrm{cm})$ postprocedure. Significant statistical comparison was seen between LVESd $(\mathrm{cm})$ pre-and post-procedure with a $\mathrm{P}$ value $<0.001$ (Figure 1 ). 


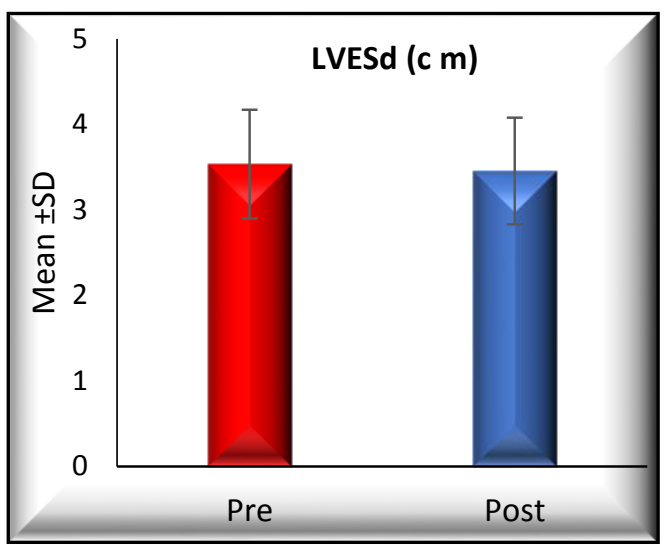

Figure (1): Comparison between LVESd $(\mathrm{cm})$ pre and post procedure.

In pre procedure, $2 \mathrm{D}$ Speckle tracking \% ranging from $-20.3 \%$ to $-10.7 \%$ with a mean of $-15.2 \pm 2.6$ SD (\%). In post procedure, $2 \mathrm{D}$ Speckle tracking ranging from $-22.4 \%$ to $-11.2 \%$ with a mean of $16.9 \pm 3.0 \mathrm{SD}(\%)$. There was significant increase in the mean 2D Speckle post procedure. There was very high statistically significant comparison between 2D Speckle tracking \% (GLS) pre and post procedure with a $\mathrm{P}$ value $<0.001$ (Figure 2).

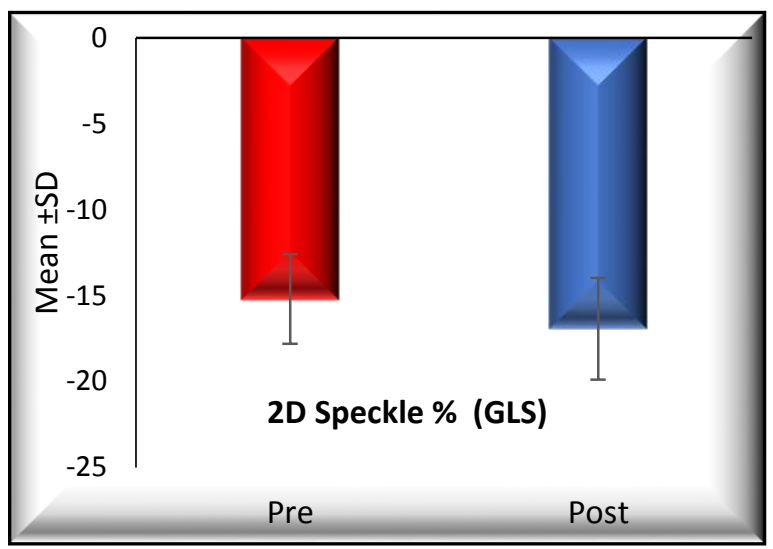

Figure (2): Comparison between 2D Speckle tracking \% (GLS) pre and post procedure. In pre procedure, LVEF (\%) by $3 \mathrm{DE}$ ranged from $42 \%$ to $72 \%$ with a mean of $56.9 \pm 7.9 \mathrm{SD}$ $(\%)$. In post procedure, LVEF (\%) by $3 \mathrm{DE}$ ranged from $46 \%$ to $74 \%$ with a mean of 59.5 $\pm 7.7 \mathrm{SD}(\%)$. There was significant increase in the mean LVEF (\%) by $3 \mathrm{DE}$ post procedure (Table 3).

Table (3): Comparison between LVEF \% by 3DE pre and post procedure.

\begin{tabular}{|c|c|c|c|c|c|c|c|c|}
\hline \multirow{2}{*}{ Time } & \multicolumn{4}{|c|}{ EF \% 3D } & \multicolumn{2}{|c|}{$\begin{array}{c}\text { Paired } \\
\text { Differences }\end{array}$} & \multicolumn{2}{|c|}{$\begin{array}{c}\text { Paired Samples } \\
\text { Test }\end{array}$} \\
\hline & Range & Mean & \pm & SD & Mean & SD & $\mathbf{T}$ & P-value \\
\hline Pre & $42-72$ & 56.883 & \pm & 7.876 & 617 & & & \\
\hline Post & $46-74$ & 59.500 & \pm & 7.675 & -2.011 & $J$ & & 01 \\
\hline
\end{tabular}

There was very high statistically significant comparison between LVEF (\%) by 3DE pre and post procedure with a $\mathrm{P}$ value $<0.001$. In pre procedure, LVEDV (ml) by 3DE ranged from $85 \mathrm{ml}$ to $155 \mathrm{ml}$ with a mean of $102.5 \pm 12.8 \mathrm{SD}(\mathrm{ml})$. In post procedure, LVEDV $(\mathrm{ml})$ by 3DE ranged from $81 \mathrm{ml}$ to $132 \mathrm{ml}$ with a mean of $96.4 \pm 10.5 \mathrm{SD}(\mathrm{ml})$. There was significant decrease in the mean LVEDV (ml) by 3DE post procedure. There was very high statistically significant comparison between LVEDV $(\mathrm{ml})$ by $3 \mathrm{DE}$ pre and post procedure with a $P$ value $<0.001$. In pre procedure, LVESV $(\mathrm{ml})$ by 3 DE ranged from $29 \mathrm{ml}$ to $90 \mathrm{ml}$ with a mean of $43.6 \pm 9.0$ $\mathrm{SD}(\mathrm{ml})$. In post procedure, LVESV $(\mathrm{ml})$ by $3 \mathrm{DE}$ ranged from $24 \mathrm{ml}$ to $69 \mathrm{ml}$ with a mean of $37.8 \pm 7.4 \mathrm{SD}$ $(\mathrm{ml})$. There was significant decrease in the mean LVESV $(\mathrm{ml})$ by 3DE post procedure. There was very high statistically significant comparison between LVEDV by $3 \mathrm{DE}$ pre and post procedure with a $\mathrm{P}$ value $<0.001$ (Figure 3).

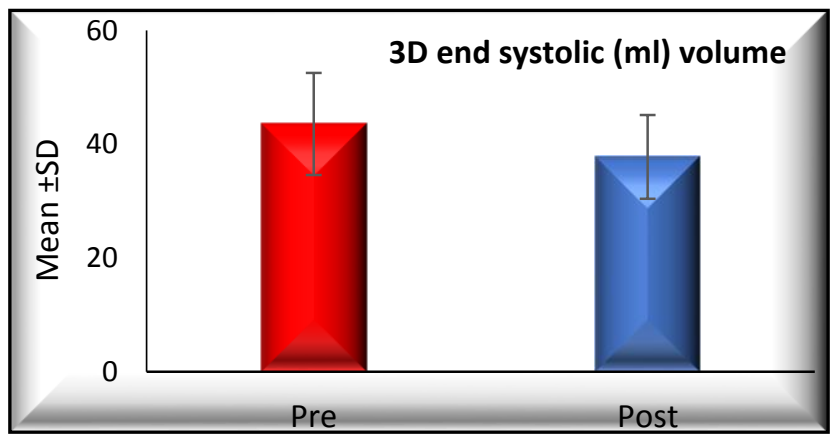

Figure (3): Comparison between LVESV(ml) by 3DE pre and post procedure. 
In pre procedure, 3D Speckle tracking $(\%)$ ranged from $-20 \%$ to $-11 \%$ with a mean of $-14.9 \pm 2.6 \mathrm{SD}(\%)$. In post procedure, 3D Speckle tracking (\%) ranged from $-22 \%$ to $-11 \%$ with a mean of $-16.5 \pm 2.8 \mathrm{SD}(\%)$. There was significant increase in the mean 3D Speckle tracking \% (LS) post procedure. There was very high statistically significant comparison between 3D Speckle tracking \% (LS) pre and post procedure with a P value $<0.001$ (Figure 4).

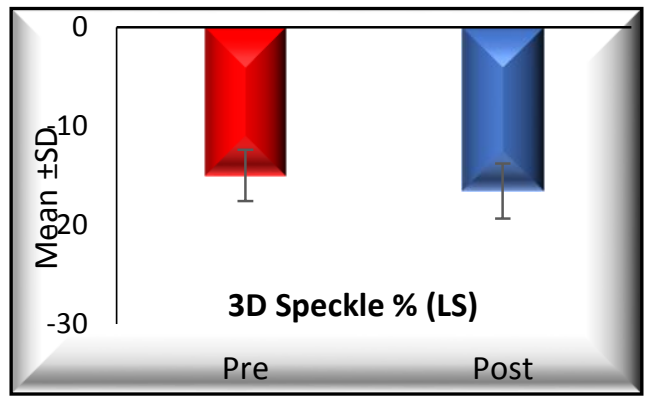

Figure (4): Comparison between 3D Speckle tracking (LS) pre and post procedure.

Multivariate analysis to detect the actual significance of individual variable in the presence of other variables: Comparison between 2D GLS \% (pre and post procedure) in diabetic patients: Diabetic patients showed improvement of 2D GLS \% with pre procedure mean of $-14.1 \pm 2.7 \mathrm{SD}$, post procedure mean of -15.4 $\pm 2.8 \mathrm{SD}$ and mean differences of $1.4 \pm 0.7$ (Table 4 ).

Table (4): Comparison between 2D GLS \% (per and post procedure) in diabetic patients.

\begin{tabular}{|c|c|c|c|c|c|c|c|c|c|c|}
\hline \multirow{2}{*}{$\begin{array}{c}\text { DM } \\
\text { (Yes) }\end{array}$} & \multicolumn{6}{|c|}{ 2D Speckle \% (GLS) } & \multicolumn{2}{|c|}{$\begin{array}{c}\text { Paired } \\
\text { Differences }\end{array}$} & \multicolumn{2}{|c|}{$\begin{array}{c}\text { Paired Samples } \\
\text { Test }\end{array}$} \\
\hline & & $\mathbf{n t}$ & & Mean & \pm & SD & Mean & SD & t & P-value \\
\hline Pre & -20.1 & 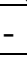 & -10.7 & -14.075 & \pm & 2.709 & \multirow{2}{*}{1.366} & \multirow{2}{*}{0.717} & \multirow{2}{*}{10.776} & \multirow{2}{*}{$<0.001 *$} \\
\hline Post & -20.9 & - & -11.7 & -15.441 & \pm & 2.781 & & & & \\
\hline
\end{tabular}

There was high statistically significant comparison between 2D GLS \% (pre and post procedure) in diabetic patients with a $\mathrm{P}$ value $<0.001$.

Comparison between 2D GLS \% (pre and post procedure) in non-diabetic patients: Nondiabetic patients showed improvement of global longitudinal strain with pre procedure mean of -16.5 $\pm 1.8 \mathrm{SD}(\%)$, post procedure mean of $-18.6 \pm 2.2$ SD $(\%)$ and mean differences of $2.1 \pm 0.8$. There was high statistically significant comparison between 2D GLS \% (pre-and post-procedure) in non-diabetic patients with a $\mathrm{P}$ value $<0.001$.

Comparison between 3D LS \% (pre and post procedure) in diabetic patients: Diabetic patients showed improvement of 2D GLS \% with pre procedure mean of $-13.8 \pm 2.6 \mathrm{SD}$, post procedure mean of $-15.2 \pm 2.6 \mathrm{SD}$ and mean differences of $1.4 \pm 0.8$. There was high statistically significant Comparison between 3D LS \% (pre -and post- procedure) in diabetic patients with a $\mathrm{P}$ value $<0.001$.

Comparison between 3D LS \% (pre and post procedure) in non-diabetic patients: Nondiabetic patients showed improvement of 3D LS \% with pre procedure mean of $-16.3 \pm 1.9 \mathrm{SD}$, post procedure mean of $-18.1 \pm 2.1 \mathrm{SD}$ and mean differences of $1.8 \pm 0.9$. There was high statistically significant comparison between 3D LS \% (pre- and post- procedure) in non-diabetic patients with a $\mathrm{P}$ value $<0.001$.

Comparison between 2D GLS (pre and post procedure) in patients with $2 \mathrm{D} E F \geq 50 \%$ : Patients with 2D EF $\geq 50 \%$ showed improvement of 2D Speckle tracking \% (GLS) with pre procedure mean of $-16.1 \pm 2.2 \mathrm{SD}(\%)$, post procedure mean of $-17.9 \pm 2.4 \mathrm{SD}(\%)$ and mean differences $1.9 \pm$ $0.805 \mathrm{SD}(\%)$. There was high statistically significant comparison between 2D GLS (pre and post procedure) in patients with $2 \mathrm{D} \mathrm{EF} \geq 50 \%$ with a $\mathrm{P}$ value $<0.001$.

Comparison between 2D GLS (pre and post procedure) in patients with 2D EF $<50 \%$ : Patients with 2D EF <50\% showed improvement of 2D Speckle tracking \% (GLS) with pre procedure mean of $-11.8 \pm 1.0 \mathrm{SD}(\%)$, post procedure mean of $-13.0 \pm 1.0 \mathrm{SD}(\%)$ and mean differences $1.2 \pm 0.8$ SD (\%). There was high statistically significant comparison between 2D GLS (pre and post procedure) in patients with $2 \mathrm{D} \mathrm{EF}<50 \%$ with a $\mathrm{P}$ value $<0.001$ (Figure 5). 


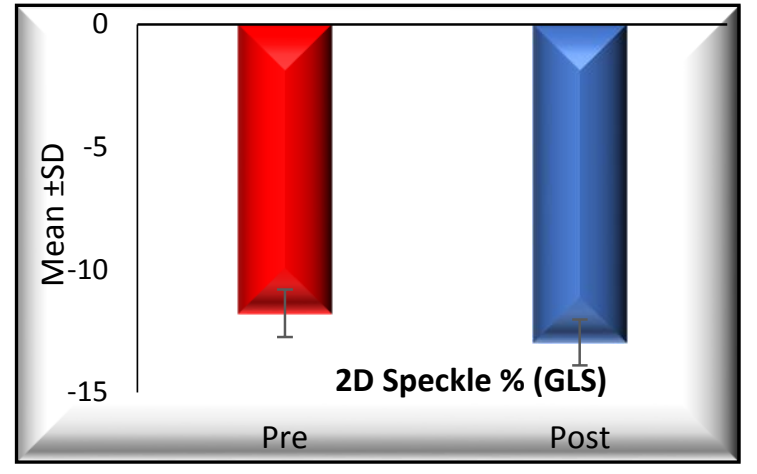

Figure (5): Comparison between 2D GLS (pre and post procedure) in patients with $2 \mathrm{D} \mathrm{EF}<50 \%$

Comparison between 3D LS (pre and post procedure) in patients with $3 \mathrm{D} \mathrm{EF} \geq \mathbf{5 0} \%$

Patients with 2D EF $\geq 50 \%$ showed improvement of 3D Speckle tracking \% (LS) with pre procedure mean of $-11.8 \pm 1.0 \mathrm{SD}(\%)$, post procedure mean of $-13.0 \pm 1.0 \mathrm{SD}(\%)$ and mean differences $1.2 \pm 0.8 \mathrm{SD}(\%)$. There was highly statistical significant comparison between 3D LS (pre and post procedure) in patients with $2 \mathrm{D} \mathrm{EF} \geq$ $50 \%$ with a $P$ value $<0.001$.

Comparison between 3D LS (pre and post procedure) in patients with 3D EF $<50 \%$

Patients with 2D EF $<50 \%$ showed improvement of 3D Speckle tracking \% (LS) with pre procedure mean of $-11.6 \pm 0.8 \mathrm{SD}(\%)$, post procedure mean of $-12.7 \pm 0.9 \mathrm{SD}(\%)$ and mean differences $1.2 \pm 0.6 \mathrm{SD}(\%)$. There was highly statistical significant comparison between 3D LS (pre and post procedure) in patients with 2D EF < $50 \%$ with a $\mathrm{P}$ value $<0.001$ (Figure 6).

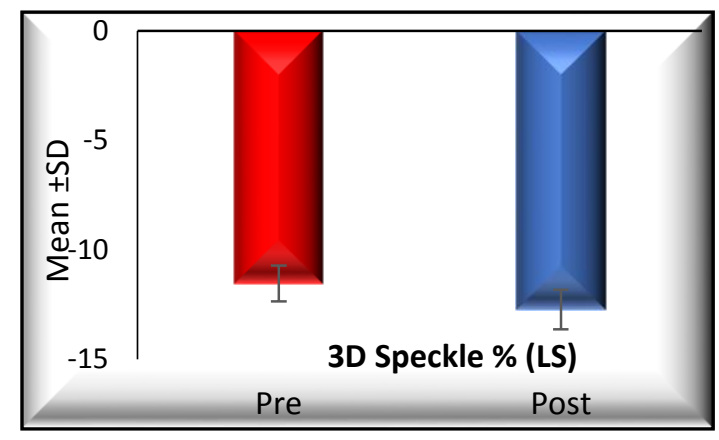

Figure (6): Comparison between 3D LS (pre and post procedure) in patients with $2 \mathrm{D} \mathrm{EF}<50 \%$

\section{Linear correlation between 2D GLS and 3D LS:}

Improvement in the 2D GLS \% was correlated with an improvement in the 3D LS \% $(r=0.9, P$ value $<0.001$ ) (Table 5) and (Figure 7).
Table (5): Linear correlation between 2D GLS \% and 3D LS \%

\begin{tabular}{|c|c|c|}
\hline \multicolumn{3}{|c|}{ Correlations } \\
\hline & \multicolumn{2}{|c|}{ 2D Speckle \% (GLS) Post } \\
\hline & $\mathbf{r}$ & P-value \\
\hline $\begin{array}{c}\text { 3D Speckle \% } \\
\text { (LS)Post }\end{array}$ & 0.983 & $<0.001 *$ \\
\hline
\end{tabular}

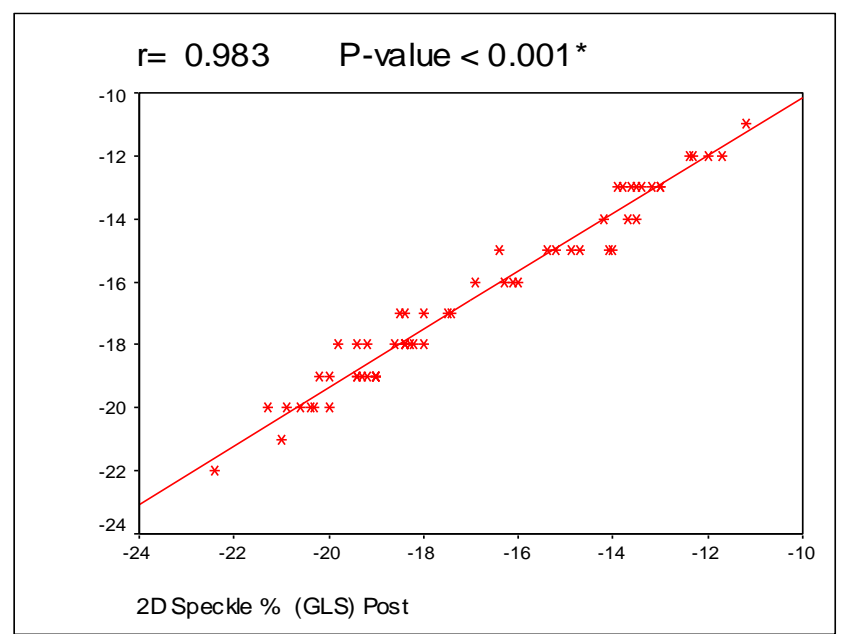

Figure (7): Linear correlation between 2D GLS \% and 3D LS\%

\section{DISCUSSION}

Erdogan et al. ${ }^{(20)}$ were the first who studied the early benefit of revascularization of CTOs (after one month) on LV function using 2D global longitudinal strain and RT3DE. They used 2DE to assess LVEF \%, LV dimensions (LVESd and LVEDd) and 2D speckle tracking (global longitudinal strain) pre- and one month after successful PCI of CTO and showed a significant increase of LVEF \% and non-significant decrease in LVESd and LVEDd. The global longitudinal strain showed a significant improvement after successful revascularization. The study used $3 \mathrm{DE}$ to assess LVEF \% and LV volume (LVESV and LVEDV) pre- and one month after successful PCI to CTO and showed a significant increase of LVEF \% and also significant decrease in LVESV and LVEDV. In our study, we added another novel echocardiographic parameter to parameters that was used by Erdogan et al. ${ }^{(20)}$. 3D speckle tracking (longitudinal strain $\%$ ) was used as a new method to assess myocardial function after PCI of a CTO. We found a significant improvement in 3D longitudinal strain after successful revascularization. In concordance with Erdogan et al. ${ }^{(20)}$ results, we found that the mean left ventricular ejection fraction (2D and 3D) significantly increased while the left ventricular end-diastolic and end-systolic volumes significantly decreased. The global longitudinal strain (2DSTE) showed a significant improvement 
after successful revascularization. In discordance with Erdogan et al. (20) results, we found a significant decrease in LVESd and LVEDd after successful revascularization.

In Wang and Ren ${ }^{(21)}$ study, improvement of LVEF was observed for follow up to 3 and 6 months after percutaneous revascularization in patients with CTO. However, by 2D speckle tracking echocardiography (2D-STE), the GLS improvement was observed as early as 1 day after CTO-PCI. These results demonstrate that 2D-STE is a reliable way to monitor early subclinical LV changes.

In the present study, our results were similar to those reported by Baks et al. ${ }^{(22)}$ who showed the beneficial effect of successful CTO revascularization on the end-systolic and enddiastolic volumes by 3DE. They showed that the extent of dysfunctional but viable myocardium before revascularization was related to an improvement in the end systolic volume and LVEF. Some studies showed that LV volume changes after MI depended on the patency status of the infarctrelated artery. When the infarct-related artery was totally occluded, a significant increase in both enddiastolic and end-systolic volumes occurred, with a resulting decrease in $\mathrm{EF}^{(25)}$.

In the present study, we found a reduction in LV volumes; however, we used RT3DE, which is more robust than 2DE. Cheng et al. ${ }^{(23)}$ used contrast-enhanced MRI to demonstrate that improved LV function and attenuated LV remodeling occurred after successful CTO PCI if the vessel patency is maintained. In another study, which used magnetic resonance imaging, early and late improvements in regional myocardial function were observed in the territory of the occlusion Kirschbaum et al. (24) and Bondarenko et al. (25) they showed the functional recovery after revascularization of hibernating myocardium on 35 patients using contrast enhanced CMR. Functional myocardial recovery started at 3-6 months with continuing improvement up to 24 months, suggesting that recovery of systolic function can be further delayed up to 24 months especially in myocardial segments with higher extent of hyper enhancement.

The improvements in LVEF \% and myocardial function in our study, based on 2DSTE, 3DSTE and RT3DE are consistent with the results of Cheng et al. ${ }^{(23)}$.

The improvements in the LVEF and GLS observed in the current study may be the result of recovered hibernating myocardium. Multiple retrospective studies showed the potential benefit of PCI in patients with CTO. Successful treatment improves LV function ${ }^{(30)}$. In a study by Ermis et al. (26) coronary intervention had a positive impact on both global and regional left ventricular function in patients with chronic coronary artery occlusion. Our results were similar to those reported by Uslu et al. (27) who showed a significant decrease in LVEDd and LVESd after PCI with significant increase in $\mathrm{EF} \%$ (two months after the procedure). However, in our study the assessment was one month after the procedure.

Magdy et al. ${ }^{(28)}$ study showed a significant reduction in LVEDd and LVESd after PCI of CTO (3 months after the procedure) and showed highly significant increase in LVEF \% after successful recanalization of CTO (3 months after the procedure).

Our results were similar to these results but the assessment of LVEDd, LVESd and LVEF \% in our study was one month after the procedure. In the present study, our results were in concordance with Megaly et al. ${ }^{(29)}$ meta-analysis of 34 studies, which demonstrated that successful CTO PCI is associated with statistically significant increase in mean LVEF by $3.8 \%$ during a mean follow-up duration of 7.9 months.

This improvement was consistent in further sensitivity and subgroup analyses. Furthermore, successful CTO PCI was associated with statistically significant reduction in LVESV indicating an improvement in LV remodeling. A previous meta-analysis of 34 studies with 2310 patients on the impact of CTO PCI on LV size and function that was performed by Hoebers et al. ${ }^{(30)}$ showed a statistically significant increase in LVEF (4.44\%) and decrease in LVEDV index (6.14 $\mathrm{mL} / \mathrm{m}^{2}$ ) as compared to baseline.

Nakamura et al. ${ }^{(31)}$ reported that ejection fraction results of a 5-center Asian registry, in which 88 patients with successfully recanalized CTOs were treated with DES, was significantly improved in these patients on follow up. our results were in concordance with Elena et al. ${ }^{(32)}$ that showed successful revascularization of CTO improves regional systolic function determined by WMSI and decreases angina. LS of treated segments and infarct size tend to decrease after procedure, with no change in global measures of cardiac function. El Shafey et al. ${ }^{(33)}$ study showed improvement in regional and global LV function measured by PW-TDI in patients with CTO vessel after successful recanalization by PCI.

Patients with DM have a greater extent of $\mathrm{CAD}$ and are prone to impaired clinical outcomes compared with non-diabetic patients. A previous observational study about CTO PCI in diabetics was performed by Safley et al. ${ }^{(34)}$ where they reported 
that DM patients do not seem to have the same survival benefits from successful PCI of a CTO as patients without DM. In our study, both patients with $\mathrm{LVEF} \geq 50 \%$ and $\mathrm{LVEF}<50 \%$ displayed significant improvement in the 2D GLS and 3D LS. In addition, diabetic and non-diabetic patients displayed significant improvement in the 2D GLS and 3D LS. In our study, we found that the improvement in the 2D GLS was correlated with an improvement in the $3 \mathrm{D}$ LS. So, use of $3 \mathrm{D} \mathrm{LS}$ is useful in assessment of global LV function and to detect the improvement of LVEF \% after successful CTO PCI.

\section{CONCLUSION}

By using novel echocardiographic techniques, our results showed that restoring the coronary blood flow in chronic total occlusion patients reduces the left ventricular volumes and dimensions, improves the left ventricular ejection fraction also improves the 2D global longitudinal strain and 3D longitudinal strain of hibernating myocardium.

\section{REFERENCES}

1- McAloon CJ, Boylan LM, Hamborg T, Stallard N, Osman F, Lim PB (2016): The changing face of cardiovascular disease 2000-2012: an analysis of the World Health Organization Global Health estimates data. Int J Cardiol., 224: 256-264.

2- Dobesh PP, Beavers CJ, Herring HR (2012): Key articles and guidelines in the management of acute coronary syndrome and in percutaneous coronary intervention: update Pharmacotherapy, 32: e348-e386.

3- Alame AJ, Karatasakis A, Karacsonyi J, Danek B, Resendes E, Parachini JR(2017): Comparison of the American College Cardiology/American Heart Association and the European Society of Cardiology guidelines for the management of patients with nonST-segment elevation acute coronary syndromes. Coron. Artery Dis., 28 (4): 294-300.

4- Brilakis E (2017): Manual of Coronary Chronic Total Occlusion Interventions: A Step-by Step Approach. 2nd ed. Cambridge, MA: Elsevier.

5- Favot M, Courage C, Ehrman R, Khait L, Levy $P$ (2016): Strain echocardiography in acute cardiovascular diseases. West J Emerg Med., 17 (1): 54-60.

6- Almeida AL, Gjesdal O, Mewton N, Choi EY, Teixido-Tura G, Yoneyama K, Lima JA (2013): Speckle-tracking pela ecocardiografia bidimensional aplicações clínicas. Rev Bras Ecocardiogr Imagem Cardiovasc., 26 (1): 38-49.

7- Smiseth AO, Torp H, Opdahl A, Haugaa KH, Urheim S (2016): Myocardial strain imaging: how useful is it in clinical decision making? Eur Heart J., 37 (15): 1196-207.

8- dos Santos NS, Vilela AA, Barretto RB, Vale MP, Rezende MO, Ferreira MC (2018): Aplicabilidade do Strain Longitudinal do Ventrículo Esquerdo na Angina Instável. Arq Bras Cardiol., 110 (4): 354-361.
9- Amundsen BH, Helle-Valle T, Edvardsen T, Torp H, Crosby J, Lyseggen E (2014): Noninvasive myocardial strain measurement by speckle tracking echocardiography: Validation against onomicrometry and tagged magnetic resonance imaging. J Am Coll Cardiol., 47 (4): 789-93.

10- Bakhoum S, Taha HS, Abdelmonem YY, Fahim M (2016): Value of resting myocardial deformation assessment by two dimensional speckle tracking echocardiography to predict the presence, extent and localization of coronary artery affection in patients with suspected stable coronary artery disease. Egypt Heart J., 3 (68): 171-179.

11- Mirea O, Duchenne J, Voigt J (2016): "Recent advances in echocardiography: strain and strain rate imaging,"F1000Research, (5): 787.

12- Lang RM, Badano LP, Tsang W, Adams DH, Agricola E, Buck T(2012): EAE/ASE recommendations for image acquisition and display using three-dimensional echocardiography. Eur Heart J Cardiovasc Imaging, 13: 1-46

13- Mizukoshi K,Takeuchi M, Nagata Y (2016): Normal values of left ventricular mass index assessed by transthoracic three-dimensional echocardiography .J Am Soc Echocardiogr., 29: 51-61.

14- Li LP, Zhang, Ran H, Dong J, Fang L, Ding Q (2017): "Evaluation of left ventricular myocardial mechanics by three dimensional speckle tracking echocardiography in the patients with different graded coronary artery stenosis. The International Journal of Cardiovascular Imaging, 33(10): 1513-1520.

15- Mancia G, Fagard R, Narkiewicz K , Redon J, Zanchetti A, Böhm M, Christiaens T, Cifkova R, De Backer G (2013): ESH/ESC Guidelines for the management of arterial hypertension: The Task Force for the management of arterial hypertension of the European Society of Hypertension (ESH) and of the European Society of Cardiology (ESC). European Heart Journal, 34 (28): 2159-2219.

16- Lang RM, Bierig M, Devereux RB, Flachskampf FA, Foster E, Pellikka PA, Picard M H, Roman MJ, Seward J, Solomon J SS, Spencer KT, Sutton M SJ, Stewart W (2006): Recommendations for chamber quantification. European Journal of Echocardiography, 7 (2): 79-108.

17- Bulwer JW, Lang RM, Bierig M, Devereux RB, Melamed R (2009): Assessment of Left Ventricular Function by Intensivists Using Hand-Held Echocardiography. J Card Fail., 15: 782-789.

18- Shimoni S, Gendelman G, Ayzenberg O, Smirin N, Lysyansky P, Edri O, Deutsch L, Caspi A, Friedman Z (2011): Differential effects of coronary artery stenosis on myocardial function: the valueof myocardial strain analysis for the detection of coronary artery disease. J Am SocEchocardiogr., 24: 748-757.

19- Altman M, Bergerot C, Aussoleil A, Einar S.Davidsen, Sibellas F, Ovize M (2014): Assessment of left ventricular systolic function by deformation imaging derived from speckle tracking: a comparison between 2D and 3D echo modalities. European Heart Journal- Cardiovascular Imaging, 15(3): 316-323. 
20- Erdogan E, Akkaya M, Bacaksiz A, Tasal A, Sönmez O, Elbey MA, Kul S, Vatankulu MA, Turfan M and Göktekin O (2013): Early assessment of percutaneous coronary interventions for chronic total occlusions analyzed by novel echocardiographic techniques. Clinics, 68 (10):1333-1337.

21- Wang P, Liu Y, Ren L (2018): Evaluation of left ventricular function after percutaneous recanalization of chronic coronary occlusions. Herz, 44(2): 170-174.

22- Baks T, van Geuns RJ, Duncker DJ, Cademartiri F, Mollet NR, Krestin GP (2006): Prediction of left ventricular function after drug-eluting stent implantation for chronic total coronary occlusions. J Am Coll Cardiol., 47 (4): 721-5.

23- Cheng ASH, Selvanayagam JB, Jerosch-Herold M, van Gaal WJ, Karamitsos TD, Neubauer S (2007): Percutaneous treatment of chronic total coronary occlusions improves regional hyperemic myocardial blood flow and contractility: insights from quantitative cardiovascular magnetic resonance imaging. JACC Cardiovasc Interv., 1 (1): 44-53.

24- Kirschbaum SW, Baks T, Van denent $M$, Sianos G, Krestin GP, Serruys PW (2008): Evaluation of left ventricular function three years after percutaneous recanalization of chronic total coronary occlusions. Am J Cardiol., 101: 179-185.

25- Bondarenko O, Beek AM, Twisk JW, Visser CA, van Rossum AC (2008): Time course of functional recovery after revascularization of hibernating myocardium: a contrast-enhanced cardiovascular magnetic resonance study. Eur Heart J., 29: 20002005.

26- Ermis C, Boz A, Tholakanahalli V, Yalcinkaya S, Semiz E, Sancaktar O (2005): Assessment of percutaneous coronary intervention on regional and global left ventricular function in patients with chronic total occlusions. Can J Cardiol., 21 (3): 275-80.

27- Uslu H, Cakmak N, Erkan ME, Hacımahmutoğlu S, Yılmaz S, Ozkan S, Sayar N (2013): Left ventricular remodeling assessment in patients with anterior acute myocardial infarction treated with successful primary percutaneous coronary intervention: an observational study .Anadolu Kardiyol Derg., 13: 675-81.

28- Magdy A, Shokry K, Foad H, Elkilany W (2017): Predictors of procedural success and improvement of left ventricular ejection fraction after successful recanalization of coronary chronic total occlusion (CTO). Egyptian Journal of Chest Diseases and Tuberculosis, $66: 377-380$

29- Megaly M, Marwan S, Peter T, Nicholas M. B, Ivan C, Mario G, Daniel L, Michael M, Anil P, Paul S, Jay T, Yale W, Louis PK, Steven MB, Emmanouil SB (2018): Meta-analysis of the impact of successful chronic total occlusion percutaneous coronary intervention on left ventricular systolic function and reverse remodeling. J Interv Cardiol., Oct;31(5):562571.

30- Hoebers LP, Claessen BE, Elias J, Dangas GD, Mehran R, Henriques JP (2015): Meta-analysis on the impact of percutaneous coronary intervention of chronic total occlusions on left ventricular function and clinical outcome. Int J Cardiol., 187: 90-96

31- Nakamura S, Muthusamy TS, Bae JH, Cahyadi YH, Udayachalerm W, Tresukosol D (2004): Impact of sirolimus-eluting stents on the outcome of patients with chronic total occlusions: multicenter registry in Asia. JACC., 43: 35A.

32- Elena RG, Susana M, Vanesa P, Jesús GM, Josebe GA, Blanca ZG, Jose D, María C, Jorge I, Javier GR (2016): Assessment of left ventricular function after percutaneous coronary intervention of chronic total occluded coronary artery by speckle tracking and cardiac magnetic resonance. JACC., 67 (13): 143.

33- El Shafey WH, Montaser SS, Badran HM, Gabr MK, Shokry KA and Galassi AR (2015): Assessment of left ventricular function before and after a percutanous coronary intervention to chronic total coronary occlusion: Doppler tissue imaging study. Menoufia Med J., 28: 400-5

34- Safley DM, House JA, Rutherford BD and Marso SP (2006): Success rates of percutaneous coronary intervention of chronic total occlusions and long term survival in patients with diabetes mellitus. Diab Vasc Dis Res., 3 (1): 45-51. 